\section{Commentary: Evidence is mounting for early intervention in asymptomatic severe aortic stenosis}

\author{
Charles M. Wojnarski, MD, MS
}

Yokoyama and colleagues ${ }^{1}$ present a meta-analysis of 8 studies comparing early surgery versus medical management in patients with asymptomatic severe aortic stenosis. Their pooled analysis of 2240 patients includes data from 1 randomized controlled trial $(\mathrm{RCT})(\mathrm{n}=145), 3$ propensity-score matched analyses $(n=780)$ and 4 observational cohort studies $(n=1315)$. Early surgery, compared with medical management, was found to have a significantly lower hazard of all-cause mortality (hazard ratio, 0.49; 95\% confidence interval, 0.36-0.68; $P<.0001)$ and cardiovascular mortality (hazard ratio, $0.42 ; 95 \%$ confidence interval, $0.22-0.82 ; P=.01$ ) at a mean follow-up of $4.2 \pm 1.3$ years.

Criteria for patient inclusion in the studies was not uniform, but at a minimum, required aortic valve mean pressure gradient $>40 \mathrm{~mm} \mathrm{Hg}$, peak aortic valve velocity $>4.0 \mathrm{~m} / \mathrm{s}$, or aortic valve area $<1.0 \mathrm{~cm}^{2}$ in patients without exertional dyspnea, syncope, presyncope, or angina. The meta-analysis is appropriately reported, consistent with Preferred Reporting Items for Systematic Reviews and Meta-Analyses standards and risk of bias was properly assessed. Notably, the 4 unadjusted studies included in the meta-analysis were at serious risk of bias due to confounding. Sensitivity analysis excluding the 4 poorer quality studies yielded similar findings to the main analysis. Overall, the meta-analytic methods are sound and heterogeneity

\footnotetext{
From the Division of Cardiovascular and Thoracic Surgery, Department of Surgery, Duke University Medical Center, Durham, NC.

Disclosures: The author reported no conflicts of interest.

The Journal policy requires editors and reviewers to disclose conflicts of interest and to decline handling or reviewing manuscripts for which they may have a conflict of interest. The editors and reviewers of this article have no conflicts of interest.

Received for publication Aug 23, 2020; revisions received Aug 23, 2020; accepted for publication Aug 24, 2020; available ahead of print Sept 6, 2020.

Address for reprints: Charles M. Wojnarski, MD, MS, Division of Cardiovascular and Thoracic Surgery, Department of Surgery, Duke University Medical Center, 2301 Erwin Rd, Durham, NC 27710 (E-mail: charles.wojnarski@ duke.edu).

J Thorac Cardiovasc Surg 2022;163:1790-1

$0022-5223 / \$ 36.00$

Copyright (c) 2020 by The American Association for Thoracic Surgery

https://doi.org/10.1016/j.jtcvs.2020.08.078
}

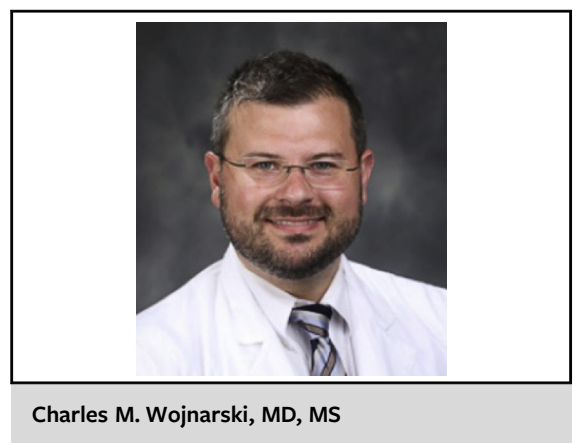

CENTRAL MESSAGE

A meta-analysis of existing litera-

ture provides level B evidence

that early surgery reduces all-

cause and cardiovascular mor-

tality in patients with asymp-

tomatic severe aortic stenosis.

of estimates acceptable; however, the quality of original evidence is limited.

Although not reported by Yokoyama and colleagues, ${ }^{1}$ a simple recalculation of their Table 1 demonstrates an average age of patients in the studies to be $68.2 \pm 7.2$ years with an average aortic valve mean pressure gradient of $51.9 \pm 4.9 \mathrm{~mm} \mathrm{Hg}$. This fairly young cohort of patients, with clearly severe aortic stenosis is very much generalizable to the patients we routinely see in consultation for determination of timing of intervention. The decision to proceed with valve replacement, weighed against the durability of existing surgical and transcatheter valves, is shared daily by patients and surgeons and should be informed by the best existing data. This meta-analysis, along with its included RCT, represents level B evidence in favor of early surgery. As the authors appropriately conclude, additional strong, prospective, and randomized data are needed before updating the medical/surgical guidelines that broadly inform practice patterns. Fortunately, 2 ongoing prospective trials are enrolling patients with asymptomatic severe aortic stenosis with randomization to either valve replacement or medical management.

The Early Valve Replacement Guided by Biomarkers of Left Ventricular Decompensation in Asymptomatic Patients with Severe Aortic Stenosis trial is randomizing patients with asymptomatic severe aortic stenosis and cardiac magnetic resonance imaging evidence of midwall (nonischemic) late gadolinium enhancement, to either surgical aortic valve replacement or medical management with a 
primary composite end point of all-cause mortality and unplanned aortic stenosis-related hospitalization at 3 years. ${ }^{2}$ The expected study completion date is October 1, 2024, with a 1000-patient enrollment.

The Evaluation of Transcatheter Aortic Valve Replacement Compared to Surveillance for Patients with Asymptomatic Severe Aortic Stenosis trial is randomizing patients with asymptomatic severe aortic stenosis, in whom transfemoral transcatheter aortic valve replacement (TAVR) is feasible, to either TAVR or medical management with a primary composite end point of all-cause death, all stroke, and unplanned cardiovascular hospitalization at 2 years. ${ }^{3}$ The expected primary study completion date is October 1, 2021, with a 1109-patient enrollment.

As we gain a better understanding of the natural history of aortic stenosis, surgical valve replacement outcomes remain outstanding, and TAVR emerging as a more palatable option to patients than surgical replacement-the concept of equipoise for an RCT comparing replacement to medical management has become more clear, as has the ability to enroll patients in such trials. We look forward to the results of the above 2 trials to provide level A evidence to firmly justify a guideline update to include a class I indication for valve replacement in patients with asymptomatic severe aortic stenosis.

\section{References}

1. Yokoyama Y, Takagi H, Kuno T. Early surgery versus conservative management of asymptomatic severe aortic stenosis: a meta-analysis. J Thorac Cardiovasc Surg. 2022;163:1778-85.e5.

2. Bing R, Everett RJ, Tuck C, Semple S, Lewis S, Harkess R, et al. Rationale and design of the randomized, controlled early valve replacement guided by biomarkers of left ventricular decompensation in asymptomatic patients with severe aortic stenosis (EVOLVED) trial. Am Heart J. 2019;212:91-100.

3. Evaluation of Transcatheter Aortic Valve Replacement Compared to SurveilLance for Patients With AsYmptomatic Severe Aortic Stenosis (EARLY TAVR). Available at: https://clinicaltrials.gov/ct2/show/NCT03042104. Accessed August 22, 2016. 